\title{
RESGATANDO AÇÕES DE ENFERMAGEM PSIQUIÁTRICA E SAÚDE MENTAL NA PRODUÇÃO CIENTÍFICA
}

\author{
Luciane Prado Kantorski ${ }^{1}$, Leandro Barbosa de Pinho ${ }^{2}$, Jacqueline de Souza ${ }^{3}$, Fernanda Mielke ${ }^{4}$
}

RESUMO: Este estudo pretende conhecer algumas ações de enfermagem psiquiátrica e saúde mental a partir de um resgate da produção científica da área. Realizou-se um levantamento da produção no período de 1980-2000 em 14 periódicos editados no Brasil conforme classificação da CAPES de 2001 da área, com nível A e B nacional e internacional. A busca resultou na seleção de 109 artigos através de uma pesquisa na base de dados do LILACS utilizando os unitermos enfermagem/psiquiátrica. Das áreas temáticas levantadas, 34 artigos trabalhavam com ações de enfermagem psiquiátrica e saúde mental. Constatamos ações resultantes do modo como a enfermagem tem-se organizado e estruturado no contexto brasileiro, e conseqüentemente, vem desenvolvendo diferentes instrumentos de sistematização da assistência na área da saúde mental. O estudo pretende problematizar a profissão de enfermagem e sua inserção na área da saúde mental como campo para produzir, discutir, sistematizar e implementar diferentes estratégias de intervenção profissional.

PALAVRAS-CHAVE: Saúde mental; Serviços de saúde; Enfermagem psiquiátrica.

\section{RESCUING PSYCHIATRIC NURSING AND MENTAL HEALTH ACTIONS IN THE SCIENTIFIC PRODUCTION}

\begin{abstract}
The study intends to find out about some actions of psychiatric and mental health nursing by rescuing the scientific production in this area. A survey was carried out among 14 journals published in Brazil between 1980 and 2000 , according to 2001 CAPES (Coordination of Qualification of Superior Level Personnel, in Portuguese) classification in the nursing area with international and national circulation, levels A and B. The search resulted in the selection of 109 articles in the LILACS database using psychiatric nursing as uniterms. From the thematic areas surveyed, 34 articles addressed psychiatric nursing and mental health actions. We verified several resulting actions from the way nursing has been organized and structured in the Brazilian context, and consequently, has been developing different systematization instruments of caring in the mental health area. The study intends to problematize nursing profession and its insertion in the mental health area while field to produce, to discuss, to systematize and to implement different strategies of professional intervention.
\end{abstract}

KEYWORDS: Mental health, Health services, Psychiatric nursing.

\section{RESCATE DE ACCIONES DE ENFERMERÍA PSIQUIÁTRICAY SALUD MENTAL EN LA PRODUCCIÓN CIENTÍFICA}

RESUMEN: Este estudio tiene El objetivo de conocer acciones de enfermería psiquiátrica y de salud mental, partiendo de un rescate de la producción científica. Se realizó una investigación de la producción científica de la enfermería en el período de 1980 hasta 2000 en 14 periódicos de Brasil, de acuerdo con la clasificación de CAPES de 2001 del área de enfermería, de circulación internacional y nacional, con nivel A y B. La recorrida resultó en 109 manuscritos a través de una investigación en LILACS, con los términos enfermería/psiquiátrica. De las áreas levantadas, 34 manuscritos trabajan con acciones de enfermería psiquiátrica y salud mental. Constatamos acciones resultantes de cómo la enfermería se arregla, se estructura en el Brasil y desarrolla diferentes instrumentos de sistematización de la atención en la salud mental. El estúdio pretende problematizar la profesión de enfermería y su inserción en el área como campo para producir, discutir, sistematizar y estructurar diferentes estrategias de intervención profesional

PALABRAS CLAVE: Salud mental; Serviços de salud; Enfermería psiquiátrica.

\footnotetext{
${ }^{1}$ Enfermeira. Doutora em Enfermagem. Professora Adjunta da Faculdade de Enfermagem e Obstetrícia da Universidade Federal de Pelotas-UFPel. Pesquisadora do CNPq.

${ }^{2}$ Enfermeiro. Doutorando em Enfermagem Psiquiátrica pela Escola de Enfermagem de Ribeirão Preto. Universidade de São Paulo-EERPUSP. Professor Assistente da Universidade Federal de Mato Grosso-UFMT.

${ }^{3}$ Enfermeira. Mestre em Enfermagem Psiquiátrica pela EERP-USP.

${ }^{4}$ Enfermeira. Mestranda em Enfermagem pela Escola de Enfermagem da Universidade Federal do Rio Grande do Sul-EEUFRGS
}

Autor correspondente

Leandro Barbosa de Pinho

Av. Antonio Joaquim, 365 - 78600-000 - Barra do Garças - MT

Recebido: 20/02/08

E-mail: lbpinho@uol.com.br

Aprovado: 01/06/08 


\section{INTRODUÇÃO}

Compreender o fenômeno da loucura exige uma complexidade de olhares. Sabemos que a loucura traz ao indivíduo uma série de mudanças em seu estilo de vida. Ela altera a dinâmica da rede de relações, importa limitações funcionais e comportamentais, demanda maior tempo, comprometimento e disponibilidade não somente de seus vínculos interpessoais, mas também dos profissionais que trabalham nos serviços de saúde e atendem, diariamente, essa clientela específica.

No entanto, se olharmos para a história da humanidade e de como ela lidou com o fenômeno da loucura, veremos que nem sempre ela foi compreendida dessa maneira. O louco, que deveria ser compreendido em suas múltiplas dimensões, acabou sendo excluído, segregado, seqüestrado, para se propor uma forma de tratamento, chamada de tratamento moral $^{(1)}$, que se baseava pouco no fortalecimento dos vínculos e muito na observação sistemática do seu comportamento, no seu estudo como objeto de intervenção médica e no controle disciplinar como prática condicionante de suas manifestações.

Essa realidade foi fruto de um contexto sóciohistórico em que se desenvolveu uma ciência médica, capaz de restabelecer relações com a loucura, mas chancelando-a como doença, observando-a para estudála, dominando-a para tratá-la. Tudo isso em prol do desenvolvimento de um corpo teórico de conhecimentos que pudessem dar conta de comprovar, cientificamente, o mecanismo do funcionamento mental ${ }^{(2)}$.

Nesse sentido, constitui-se toda uma gama de modalidades de interpretação organicistas para o fenômeno mental. No campo da saúde, a psiquiatria estrutura-se como conhecimento científico, propondo tratá-la na medida em que a considera um desarranjo funcional do organismo. No terreno social, nasce uma concepção sociogênica da loucura, que pretendia explicar, numa relação de causa-efeito, o desenvolvimento da sociedade e o aparecimento de desordens mentais. Como expressão de periculosidade social e incentivado por um sistema político-econômico capitalista, o louco ficou conhecido como alguém que desvia da ordem, sendo impedido de ter acesso a funções sociais, como educação, cultura, lazer e trabalho ${ }^{(3)}$.

A temática da loucura tem sido focalizada sob diferentes ângulos no contexto atual de reestruturação das práticas psiquiátricas. Por meio da transformação do olhar que desenvolvemos sobre o fenômeno da loucura por meio do movimento da reforma psiquiátrica $^{(4)}$, vem-se ressignificando o modo como operamos com ela, com o louco e as relações destes com a sociedade. Nesse sentido, as diversas profissões da saúde, entre elas a enfermagem, passaram a acompanhar essa tendência, com a incorporação de diferentes saberes e práticas em seu processo de trabalho.

Dessa forma e tendo por base o exposto acima, o presente estudo pretende conhecer ações de enfermagem psiquiátrica e saúde mental desenvolvidas pelos enfermeiros, a partir de um resgate da produção científica da área no período de 1980-2000.

\section{A TRAJETÓRIA METODOLÓGICA}

Consiste num estudo bibliográfico, no qual realizou-se um levantamento da produção científica de enfermagem de 1980 a 2000 em 14 periódicos editados no Brasil conforme classificação da CAPES de 2001 da área de enfermagem de circulação internacional e nacional, com nível A e B.

O referencial teórico-metodológico escolhido para o estudo foi proposto por Merhy ${ }^{(5)}$, que divide as tecnologias de atenção em saúde em duras, leve-duras e leves. As tecnologias duras são aquelas em que se utiliza máquinas e equipamentos como auxiliares na assistência em saúde. As tecnologias leve-duras são entendidas como aquelas em que ocorre a união dos processos de trabalho com os saberes de cada especialidade, resultando em um saber estruturado, como a clínica médica, psiquiátrica e outras. Já as tecnologias leves são consideradas pelo autor como tecnologias de saber não estruturadas, que estão em processo de atualização, e que medeiam as relações entre profissional e paciente, como o vínculo, o acolhimento, o relacionamento terapêutico e outras.

A busca resultou na seleção de 109 artigos através de uma pesquisa na base de dados do LILACS utilizando os unitermos enfermagem/psiquiátrica. Dos 14 periódicos analisados, 04 (quatro) não apresentaram produção científica na área. Tivemos acesso aos 109 artigos e procedemos a uma classificação por periódico, período e área temática. Das áreas temáticas levantadas, 34 artigos trabalhavam com ações de enfermagem psiquiátrica e saúde mental.

A sistematização desses artigos foi realizada conforme o campo de conhecimentos em que se focam as referidas ações de enfermagem psiquiátrica e saúde mental (Quadro 1). Neste estudo, discutimos as ações 
de enfermagem psiquiátrica focadas no hospital psiquiátrico (10 artigos no total) e nos serviços comunitários (8 artigos no total) como campo de saberes e práticas do enfermeiro.

Quadro 1-Ações de enfermagem psiquiátrica e saúde mental nos diferentes contextos de atuação do enfermeiro

\begin{tabular}{|lc|}
\hline \multicolumn{1}{|c}{ LOCAL DAS AÇÕES DE } & NÚMERO \\
ENFERMAGEM PSIQUIÁTRICA E & DE \\
SAÚDE MENTAL & ARTIGOS \\
Hospital Psiquiátrico & 10 \\
Hospital Geral & 5 \\
Hospital Dia & 1 \\
CAPS/ NAPS & 2 \\
Ambulatório & 3 \\
Unidade Básica de Saúde & 6 \\
Artigos que abordam diferentes ações de & 7 \\
enfermagem psiquiátrica & \\
TOTAL & 34 \\
\hline
\end{tabular}

\section{RESULTADOS E DISCUSSÕES}

Procuramos dividir os resultados de acordo com o campo de práticas do enfermeiro, para facilitar as reflexões. Iniciamos com a discussão sobre as ações do enfermeiro no contexto hospitalar, mais precisamente no hospital psiquiátrico. Nessa primeira situação, encontramos 10 artigos que abordam o assunto.

O primeiro estudo foi desenvolvido num hospital psiquiátrico, objetivando planejar, treinar e avaliar a equipe de enfermagem na utilização de contenção nas emergências psiquiátricas. Neste treinamento, o enfermeiro ficava responsável pelo planejamento, execução e avaliação do uso das técnicas de contenção. As modalidades desenvolvidas durante o treinamento foram a contenção verbal, química e mecânica. Na primeira, considera-se o uso de manejo verbal, firme e amigável. Na segunda, caso a primeira não tenha sucesso terapêutico, utiliza-se a medicação. Na contenção mecânica, analisa-se o fracasso das duas primeiras modalidades, para depois discutir a necessidade do uso da eletroconvulsoterapia, da insulinoterapia ou até mesmo da contenção cirúrgica (lobotomia). As contenções verbais, químicas e mecânicas são realizadas por todos os funcionários de enfermagem e supervisionadas pelo enfermeiro ou médico psiquiatra ${ }^{(6)}$.

Em outro estudo, houve adaptação dos padrões de assistência de enfermagem elaborados por enfermeiras norte-americanas para servir de parâmetro na avaliação da assistência ao doente mental no contexto brasileiro. Os critérios de avaliação constam de quatorze itens, submetidos à apreciação de enfermeiros que atuam em Hospital Psiquiátrico, para posterior implementação. Segundo ele, foram descritas como atividades prioritárias do enfermeiro, as atividades de recepção do paciente, verificação de sinais vitais e demais avaliações do estado físico do indivíduo. Além disso, ressalta-se a necessidade do enfermeiro em conhecer suas atribuições como coordenador, planejador e avaliador da assistência de enfermagem, assumindo tanto seu papel de orientador, quanto seu papel de mediador dentro da instituição hospitalar ${ }^{(7)}$.

Em outro trabalho, abordou-se a importância da percepção, da observação e da anotação do enfermeiro no atendimento ao paciente em um Hospital Psiquiátrico. Durante a análise, ficou evidenciado o papel do enfermeiro como punidor e repressor, orientando seu trabalho não para atender as necessidades dos pacientes, como ele se propõe, mas para responder a interesses médicos, institucionais e a seus próprios, deixando por último, os interesses do paciente ${ }^{(8)}$.

Através de observações em Hospitais Psiquiátricos, outro estudo discute que tanto os membros da equipe de enfermagem como os estudantes têm dificuldades em prestar assistência a pacientes psicóticos. As atividades do enfermeiro se atêm à observação sistemática e contínua de seu comportamento, como descrição das manifestações, proteção contra o risco do suicídio, cuidados de higiene e conforto, observação do sono-vigília e socialização gradual. Ressaltou-se que estas atividades não serão efetivas caso não seja levada em conta à individualidade de cada paciente e a participação no planejamento e na implementação do plano para sua recuperação ${ }^{(9)}$.

No próximo estudo, o objetivo era o de poder compreender as necessidades de uma cliente internada em uma enfermaria psiquiátrica de um Hospital Psiquiátrico. Com ele, afirmou-se que a aproximação de cada um dos membros da equipe de enfermagem com a clientela internada dar-se-á a partir de uma efetiva supervisão da enfermeira. E que, a esta cabe subsidiar esta equipe tecnicamente, com o objetivo de aprimorá-la, propiciando condições favoráveis para um adequado desenvolvimento das atividades assistenciais. A enfermeira psiquiátrica também deve assumir a responsabilidade da educação permanente da equipe de enfermagem, no intuito de produzir sujeitos mais 
qualificados para o atendimento ao doente mental ${ }^{(10)}$.

Pode-se considerar como marco fundamental do nascimento da enfermagem moderna no mundo a ascensão do sistema capitalista europeu e a estruturação do conhecimento médico institucionalizado, juntamente com a decadência dos sistemas monástico-caritativos que detinham o saber sobre a saúde e a doença entre os séculos XVI e XIX. Esse contexto foi favorável ao redimensionamento das práticas empíricas da enfermagem, antigamente exercidas por mulheres nos ambientes domésticos, para culminar na ascensão de um novo conhecimento, desta vez científico, elevando a enfermagem à categoria de profissão ${ }^{(11)}$.

No caso da enfermagem psiquiátrica, o conhecimento desenvolvido por Florence Nightingale sobre a organização e o saneamento dos espaços hospitalares, com práticas disciplinares, foi determinante na redefinição do próprio papel do enfermeiro no hospital psiquiátrico. Um exemplo da importância deste profissional nesse contexto está no papel do enfermeiro Pussin em seus constantes trabalhos como colaborador de Pinel nas intervenções sobre o sofrimento mental em Bicêtre e Salpetrière ${ }^{(1)}$.

A psiquiatria e a enfermagem psiquiátrica são oriundas do conhecimento desenvolvido sobre o hospital psiquiátrico e no hospital psiquiátrico. O hospício era a instituição que visava a disciplinar os corpos, com práticas pedagógicas baseadas na continência e a autoridade. Os trabalhadores da enfermagem tornaram-se coadjuvantes das práticas de intervenção sobre o fenômeno da loucura, sendo, nesse sentido, executores da ordem disciplinar partida dos médicos psiquiatras da época e consolidadas no espaço do hospício ${ }^{(12)}$.

Compreendemos que a prática do enfermeiro, centrada no hospital psiquiátrico e ressaltada nos estudos anteriores, de certa forma evidencia uma especificidade conquistada historicamente pela profissão em termos de atribuições profissionais. No hospital psiquiátrico, as atividades administrativas, voltadas para a organização, saneamento dos espaços e disciplinarização dos corpos, também desvelam um conhecimento específico incorporado pela enfermagem sobre a intervenção no processo saúdedoença. Uma tecnologia leve-dura, que se embasa em saberes estruturados para organizar-se como conhecimento e prática profissional, voltados para o reconhecimento dos determinantes biológicos do adoecimento mental e reproduzido, na realidade assistencial, por meio de práticas institucionalizantes sobre o comportamento e as manifestações mentais.

Tal especificidade profissional se estratificou e se fortaleceu coincidentemente nas relações de poder complexas que eram estabelecidas entre o louco, o enfermeiro psiquiátrico e o médico psiquiatra. Relação esta que atualmente, num contexto de transformação da assistência psiquiátrica, tem buscado a inversão dos saberes sobre o corpo para valorizar o saber sobre o sujeito, baseando-se na crítica às estruturas cristalizadas de conhecimento e construindo novas possibilidades de atuação, centralizadas no princípio da horizontalidade, da autonomia, da temporalidade, das necessidades, dos desejos, das trocas e das possibilidades.

Parte dessas nossas inferências vai ao encontro dos achados dos outros 5 (cinco) estudos, sendo os 03 (três) primeiros abordando o hospital psiquiátrico como campo de ações de enfermagem psiquiátrica e as dificuldades enfrentadas pelo enfermeiro em sua realidade assistencial e os 2 (dois) últimos enfocando a transformação das práticas de enfermagem no contexto hospitalar psiquiátrico.

No primeiro, buscou-se identificar através dos discursos dos enfermeiros de um hospital psiquiátrico da região oeste do Paraná, o que eles compreendem de enfermagem psiquiátrica e como tem sido sua prática. Após a análise dos dados as autoras concluíram que os enfermeiros possuem dificuldades em definir seu papel ao atuar em psiquiatria e por isto acabam exercendo funções administrativas, sendo este um papel mais definido e esperado por parte da instituição ${ }^{(13)}$.

No seguinte, destacou-se que a atuação de enfermeiros psiquiátricos em um macro-hospital psiquiátrico estatal de São Paulo, ressaltando a problemática que as práticas da enfermagem psiquiátrica enfrentam no cotidiano dessas instituições. De acordo com o estudo, a maioria dos funcionários trabalha insatisfeita, há um número exagerado de pacientes e falta pessoal qualificado para atender, com precárias interações terapêuticas entre pacientes e profissionais. Além disso, analisou-se que a relação enfermeiro-paciente limitava-se às queixas (clínicas ou psiquiátricas), fato esse responsável por impossibilitar a prática efetiva da enfermagem na compreensão dos problemas do paciente nessas instituições ${ }^{(14)}$.

Analisou-se, em outro estudo, o papel do enfermeiro psiquiátrico na assistência ao doente mental internado em dois macro-hospitais psiquiátricos: um público e outro privado. Constatou-se que, nos hospitais investigados, o enfermeiro dedica-se 
prioritariamente à organização administrativa do espaço de tratamento, sendo responsável pelas ações da equipe de enfermagem, pelo controle das escalas e as faltas de pessoal. Também considerou-se que o enfermeiro, nessas condições, ocupa um espaço intermediário contraditório, pois é submisso e autoritário ao mesmo tempo (submisso ao médico e autoritários ao delegar atividades aos auxiliares e técnicos de enfermagem $)^{(15)}$.

No próximo estudo, é relatada uma experiência ocorrida no instituto de psiquiatria da UFRJ, onde se iniciou um processo de transformação no atendimento clínico, através da tentativa de mudar o papel da enfermagem psiquiátrica e terapia ocupacional. Após oito anos de experiência clínica com teatro terapêutico, observou-se ser de suma importância para transformar a dinâmica institucional, mudar o papel do enfermeiro psiquiátrico, tradicionalmente centrado na vigilância e repressor, evoluindo para um papel de terapeuta, compreensivo e atuante na dinâmica do paciente. Sendo o objetivo final do trabalho encontrar resoluções para o impasse institucional em que se encontra o tratamento do doente mental ${ }^{(16)}$.

Outro estudo relatou uma experiência no instituto de psiquiatria da UFRJ sobre a transformação do papel institucional do enfermeiro psiquiátrico, através do questionamento de papéis, rotina, métodos repressivos e democráticos de intervenção no comportamento utilizados no interior da instituição, além da relação de poder no tratamento dos pacientes. Tal estudo buscou ativar o papel terapêutico do enfermeiro e, em conseqüência, mudar o papel institucional e assim proporcionar um melhor tratamento ao cliente. Segundo ele, a enfermagem caminha no sentido de encontrar sua verdadeira função e de tirar de si outras funções, como as burocráticas, atribuídas pela instituição e que não se justificam do ponto de vista terapêutico ou científico ${ }^{(17)}$.

As implicações da prática do enfermeiro psiquiátrico, juntamente com as contradições que nasceram em torno de seu saber profissional nesse contexto, são fatores que possibilitam re-discutir o processo de trabalho nesses espaços sociais. No caso da enfermagem psiquiátrica, a segregação do indivíduo em sofrimento mental, como já discutido, foi o resultado da materialização de mecanismos de intervenção voltados para a compreensão da estrutura fisiológica das manifestações da loucura. Entendemos que esse saber, reproduzido pelo enfermeiro em suas tendências disciplinares sobre os espaços sociais e corpos de indivíduos e coletivos, parece esbarrar num eixo contraditório que produz um deslocamento de saberes sobre o sujeito para a doença. Isso pode ter produzido repercussões no modo de operar com o saber instituído, no modo de organizar o serviço de saúde para atender a clientela específica, no modo de organizar as práticas de enfermagem e da equipe e, conseqüentemente, no modo de assistir e compreender o sujeito como um todo.

Vale lembrar que a enfermagem é uma profissão ainda recente e que busca pari passu a transformação de seus saberes e práticas. No entanto, a referida transformação só é feita no decorrer da história, com o apontamento das contradições, com o exercício cotidiano da superação dialética, a mobilização daquilo que pensamos ser "cristalizado" e "posto", o preenchimento das lacunas, o entrelaçamento de esforços. Afinal, cuidar não é somente valorizar objetos, papéis ou rotinas. Cuidar é também valorizar a dimensão complexa do outro, a dinamicidade, o espaço de trocas, as relações interpessoais, a liberdade de ir e vir, o “devir”, os vínculos sociais.

A seguir, passamos a discutir as ações de enfermagem psiquiátrica nos serviços comunitários como novo campo de saberes e práticas do enfermeiro.

\section{Ações de Enfermagem Psiquiátrica nos Serviços Comunitários}

As ações de enfermagem psiquiátrica parecem ser, nos serviços comunitários, mais intrigantes e complexas do que no hospital psiquiátrico. Dos artigos analisados, 6 (seis) destacam as implicações dessas ações no contexto das unidades básicas de saúde, enquanto 2 (dois) discorrem sobre a prática do enfermeiro nos serviços substitutivos de saúde mental.

No primeiro, discute-se a concretização de um programa realizado em Guiné-Bissau, cujo objetivo era capacitar enfermeiros e agentes principais do programa básico de saúde mental para diagnosticar e tratar pacientes com epilepsia e psicóticos. Durante doze seminários desenvolvidos no período de uma semana, os enfermeiros receberam uma formação básica para a atuação no campo da saúde mental ${ }^{(18)}$.

No próximo, relatou-se uma experiência de alunos e professores de enfermagem psiquiátrica numa sala de espera de um Sanatório de Goiás. Como propósito, discutia-se a implantação de um atendimento de enfermagem nos serviços de saúde com base no levantamento das necessidades em saúde mental. Entre as atividades-alvo estavam palestras voltadas para 
a educação em saúde, observação do comportamento através de atividades recreativas em grupo. Constatouse que a estratégia de atendimento na sala de espera reduziu o stress e a ansiedade dos usuários, sendo também uma oportunidade de estabelecer trocas por meio das relações interpessoais ${ }^{(19)}$.

Num relato de experiência vivenciado por alunos de graduação em enfermagem do oitavo semestre numa unidade básica de saúde de Belém-PA, discutiuse que as ações programáticas atendiam a comunidade nos níveis primários e secundários. No programa de saúde mental havia consulta médica para pacientes novos e revisão para pacientes antigos. Levando em consideração o referencial teórico da psiquiatria preventiva, foi proposto o atendimento em grupo. Os acadêmicos atendiam em diversos programas, como no de pré-natal, de doenças transmissíveis, de prevenção de câncer de colo uterino e no programa de atendimento a adolescentes, em escolas da região realizando ações de saúde mental integradas a estes. O trabalho obteve resultados positivos e os alunos passaram a valorizar a prevenção como estratégia de cuidado em saúde mental ${ }^{(20)}$.

No estudo seguinte, discutiu-se, na forma de um relato de experiência ocorrido na UBS em BelémPA, a saúde mental na atenção primária. A experiência deu-se com uma cliente portadora de câncer de colo uterino e vinculada ao programa de saúde da mulher. Na ocasião do diagnóstico da enfermidade, acadêmicos de enfermagem passaram a fazer visitas domiciliares a esta paciente, orientando-a a fazer novos exames. Nessa oportunidade, descobriu-se que as lesões uterinas poderiam ser cauterizadas, sem necessidade de cirurgia. Com base nas visitas domiciliares e na atenção dos alunos, houve melhora progressiva do quadro depressivo da paciente, evitando outras modalidades de intervenção(21).

Em outros dois estudos, refletiu-se sobre o papel desempenhado por enfermeiros psiquiátricos na assistência a pessoa com sofrimento psíquico, bem como de pessoas com problemas orgânicos que também apresentam problemas emocionais. Abordouse ainda a atuação dos enfermeiros em programas e grupos, atendendo hipertensos, alcoolistas, mulheres no climatério e pacientes submetidas a procedimentos cirúrgicos. Destacou-se também o enfermeiro atuando em programas ligados à saúde da criança, nos quais ele trabalha aspectos educativos com os pais, como as mudanças provocadas pelo desenvolvimento ${ }^{(22-23)}$.

Os apontamentos feitos nos estudos anteriores têm ressaltado a atenção primária como novo campo de saberes e práticas da enfermagem no campo da saúde mental. Vale ressaltar que essa realidade foi possível mediante a complexa transformação pela qual as políticas públicas de saúde no Brasil passaram nos últimos 20 anos. Com o desenvolvimento da consciência sanitária a partir do movimento da reforma sanitária( $^{(24-25)}$, elevou-se o foco na atenção primária em contraposição a priorização histórica do modelo biomédico-hospitalocêntrico institucionalizante. Ampliou-se também o conceito de saúde, o qual passa a ser reflexo do modo de viver dos sujeitos, com seus determinantes biológicos, psicológicos, culturais, econômicos e sociais.

Os mesmos estudos apresentados, ainda que concentrados nas discussões no campo da saúde mental, trazem a incorporação de uma gama de novas tecnologias de cuidado às ações de enfermagem. Destacam-se o acolhimento e o vínculo como tecnologias leves que balizam uma concepção ampliada do processo saúde-doença, assim como seus instrumentos facilitadores (visitas domiciliares, atendimento em grupo, sala de espera e palestras), que buscam reconhecer as diversas dimensões da vida, considerando necessidades do sujeito, da família e do território onde ele vive. Fazem também uma interlocução da saúde mental com as diferentes fases do ciclo de vida do sujeito, como a saúde da mulher e a saúde da criança. Ou seja, deslocar novamente nosso olhar sobre as tecnologias estruturadas e centradas na doença para as tecnologias leves que se fundamentam na completude do sujeito, em que acolher, formar vínculos, valorizar contextos e temporalidades são mais importantes do que conceitos aprisionados, papéis, rotinas, condicionamentos e vigilância.

É nesse contexto que se situam os Centros de Atenção Psicossocial (CAPS). Nascidos com o compromisso de serem substitutivos ao manicômio, operam de forma regionalizada no território geográfico e existencial onde vive o sujeito em sofrimento mental, para que este seja acompanhado em sua comunidade e possa receber cuidados baseados na prevenção, promoção, reabilitação e tratamento ${ }^{(26)}$. Diante dessa nova realidade no campo da saúde mental destacamse 2 (dois) estudos que abordam as ações do enfermeiro psiquiátrico nesses serviços.

No primeiro, desenvolvido num Centro de Atenção Psicossocial do Estado do Rio Grande do Sul, objetivou-se desenvolver um instrumento de sistematização do cuidado em enfermagem psiquiátrica 
baseado no referencial teórico-metodológico da Reforma Psiquiátrica e no processo de enfermagem segundo Irving. Propôs-se a construção de um projeto terapêutico individualizado ao portador de sofrimento psíquico, inserindo-o num leque interdisciplinar de possibilidades de reabilitação psicossocial. As autoras concluem que o projeto terapêutico, abordado de maneira multifocal, produz sujeitos, possibilita prestar assistência integral e ética, assim como permite o resgate da cidadania do portador de sofrimento mental ${ }^{(27)}$.

Enquanto o primeiro estudo buscou trabalhar diretamente com o portador de sofrimento mental, o segundo estudo ${ }^{(28)}$ centrou-se na estrutura de funcionamento de um serviço. Em pesquisa num Centro de Atenção Psicossocial (CAPS) do interior do Ceará, constatou-se que a maioria dos procedimentos, como consultas e triagens, era realizada pelo enfermeiro, pois, muitas vezes, o psiquiatra não se fazia presente. Após a análise dos dados, as autoras concluem que algumas dificuldades ainda rondam o interior desses serviços e ameaçam sua sobrevivência, como a excessiva clientela para atendimento, o reduzido número de profissionais e a precarização das relações trabalhistas.

Os CAPS são serviços estratégicos de atendimento em saúde mental e primordiais na consolidação do processo de reforma psiquiátrica brasileira. Atendem a uma concepção regionalizada de produção de saúde e fazem parte integrante de uma rede complexa de serviços designada a contemplar as diferentes dimensões do indivíduo em sofrimento mental. O caráter dialético desses serviços desvela-se como concretude no tempo, possivelmente capaz de desfazer os "vícios do manicômio" e transformar a internação em autonomia, a exclusão em inserção, a assistência em acolhimento e o tratamento em cuidado.

No entanto, mesmo os CAPS construindo-se como novas modalidades de intervenção ao fenômeno da loucura, as discussões sobre a reforma psiquiátrica ainda precisam avançar no contexto brasileiro. Alguns estudos $^{(29-30)}$ têm discutido o assunto. Ressaltaram-se algumas contradições, como o uso do mecanismo disciplinar de poder sobre o outro, contrastando liberdade e tutelarização, culpabilizando as famílias, normalizando os serviços, tornando-o repleto de rotinas, geralmente rígidas. Além do incentivo dos gestores municipais no compromisso de perceber o sofrimento mental como parte integrante dos problemas de saúde da região, preferindo afastá-los ao invés de incluí-los no rol das políticas públicas locais.
Devemos ressaltar que os CAPS, embora apresentem algumas dificuldades operacionais no contexto de cuidados em saúde mental, são serviços recentes, que vêm a substituir todo um aporte de saberes cristalizados há séculos no e pelo manicômio. Acredita-se que os CAPS possam, de modo funcionante, reaproximar sujeitos, humanizar as relações e possibilitar o exercício cotidiano da superação dos obstáculos. Além disso, considera-se que este modo de cuidar possa deslocar um conhecimento estruturado em tecnologias cristalizadas para contemplar o sujeito e suas experiências vivenciadas, possuidor de demandas, relações, conhecimentos, podendo e devendo ser assistido, inserido, tratado, mas antes de tudo, cuidado. Essas e outras experiências vêm sendo constantemente ressaltadas na produção científica da área atualmente, sendo referência para a construção-reconstrução de novas tecnologias de cuidado nesse campo tão complexo como é a saúde mental.

\section{CONSIDERAÇÕES FINAIS}

Esse estudo buscou conhecer, na literatura do campo da saúde mental, diferentes ações de enfermagem psiquiátrica e de saúde mental sob a loucura no período de 1980-2000. Constatamos as diversas ações resultantes do modo como a enfermagem foi se organizando e se estruturando no contexto brasileiro, e conseqüentemente, desenvolvendo diferentes instrumentos de sistematização da assistência na área da saúde mental.

Com base no hospital psiquiátrico, destacamos a participação do enfermeiro no planejamento, sistematização, organização, supervisão e treinamento dos profissionais de enfermagem. Além disso, a utilização de modalidades de intervenção ao fenômeno da loucura concentradas no olhar vigilante, no poder disciplinar e nos mecanismos de repressão ao comportamento do louco.

No que refere aos serviços comunitários, pudemos notar a ampliação das ações de enfermagem psiquiátrica e saúde mental, com o foco em tecnologias leves, centradas no saber relacional. Destacam-se o acolhimento, o vínculo com usuário e famílias, a organização da intervenção no território, além da participação do enfermeiro nas atividades de educação em saúde, voltadas para as diversas fases do ciclo vital (infância, adolescência, fase adulta e maturidade). Já nos CAPS, embora ainda permeados de 
contradições por serem serviços relativamente recentes no contexto brasileiro, há uma teia de possibilidades de atuação do profissional de enfermagem, que vai desde o acolhimento até a participação política nas decisões que envolvam o campo da saúde mental.

O recorte da produção científica por nós realizado pretende problematizar a profissão de enfermagem, tomando como foco as tecnologias de cuidado em saúde mental, área esta em constante transformação, para que possamos produzir, discutir, sistematizar e implementar diferentes estratégias de intervenção profissional.

\section{REFERÊNCIAS}

1. Foucault M. História da Loucura. $8^{\mathrm{a}}$ ed. São Paulo: Perspectiva; 2003.

2. Pessotti I. A loucura e as épocas. Rio de Janeiro: 34; 1994.

3. Birman J. A psiquiatria como discurso da moralidade. Rio de Janeiro: Graal; 1978.

4. Amarante P. A clínica e a Reforma psiquiátrica. In: Amarante P, ed. Archivos de saúde mental e atenção psicossocial 1; Rio de Janeiro, Brasil. Rio de Janeiro: Nau Editora; 2003. p.45-65.

5. Merhy EE. Em busca da qualidade dos serviços de saúde: os serviços de porta aberta para a saúde e o modelo tecnoassistencial em defesa da vida (ou como aproveitar os ruídos do cotidiano dos serviços de saúde e colegiadamente reorganizar o processo de trabalho na busca da qualidade das ações de saúde). In: Cecílio LCO, ed. Reinventando a mudança na saúde. Saúde em Debate - Série Didática; 1997. p.117-60.

6. Augustin MRGL, Augustin PV. Rotinas de emergências psiquiátricas. Rev Gaúcha Enferm. 1986; 7(2):314-24.

7. Arantes EV, Stefanelli MC, Fukuda IMK, Forcella HT. Estudo preliminar sobre padrões de assistência de enfermagem psiquiátrica. Rev Bras Enferm. 1984; 37(3/ 4):205-17.

8. Fraga MNO, Araújo MV, Goulart SF. Influência das percepções, observações e anotações do enfermeiro sobre as ações da equipe de saúde no atendimento ao paciente psiquiátrico. Rev Bras Enferm. 1980; 33:223-35.

9. Teixeira MB, Barros S. Assistência de enfermagem a pacientes com manifestação de comportamento decorrente de alheamento da realidade. Rev Esc Enferm USP. 1991; 25(3):335-46.
10. Branco ALC, Tocantins FR, Elsas BX, Rodrigues BMRD. Avaliação de enfermagem: necessidades de cliente internada em enfermaria psiquiátrica e a assistência de enfermagem. Esc Anna Nery Rev Enferm. 1998; 2(1/2):93-100.

11. Silva GB. Enfermagem profissional: análise crítica. São Paulo: Cortez; 1986.

12. Oliveira AGB, Alessi NP. O trabalho de enfermagem em saúde mental: contradições e potencialidades atuais. Rev Latino-Am Enferm. 2003; 11(3):333-40.

13. Rodrigues RM, Schneider JF. A enfermagem na assistência ao indivíduo em sofrimento psíquico. Rev Latino-Am Enferm. 1999; 7(3):33-40.

14. Baldio VM, Silva GB. Aatuação do enfermeiro psiquiátrico em um macro-hospital psiquiátrico estatal: análise de uma experiência. Rev Baiana Enferm. 1994; 7(1/2):84-91.

15. Filizola CLA. O papel do enfermeiro psiquiátrico oprimido e opressor. Rev Esc Enferm USP. 1997; 31(2):173-90.

16. Filho CJC, Araújo FA. Transformação da instituição psiquiátrica - um caminho (I). J Bras Psiquiatr. 1986; 35(5):313-8.

17. Costa AZ, Maciel AM, Elizêu MC. A enfermagem na psiquiatria - uma transformação institucional. J Bras Psiquiatr. 1983; 32(2):115-20.

18. Jong JTVM, Oostrom BV. Avaliação de um programa básico de saúde mental na Guiné-Bissau. Jor Bras Psiquiatr. 1991; 40(11):31-4.

19. Esperidião E, Oliveira MAE, Pontiere MSS. Sala de espera: uma ocasião de atenção primária em saúde mental relato de experiência. Rev Bras Enferm. 1992; 45(2/3):145-8

20. Silva WV.Atendimento de enfermagem em saúde mental em unidade básica de saúde. Rev Enferm UERJ. 1998; 6(1):277-84.

21. Silva WV. Assistência de enfermagem em saúde mental: relato de experiência. Acta Paul Enferm. 1996; 9(2):47-50.

22. Barros S, Forcella HT, Teixeira MB, Arantes EC. Ações de saúde mental do enfermeiro em unidade básica de saúde. Rev Esc Enferm USP. 1987; 21(3):255-62.

23. Barros S. Trabalho da equipe multiprofissional em saúde mental em centros de saúde: contribuição do enfermeiro. Rev Esc Enferm USP. 1987; 21(3):221-4.

24. Mendes EV. Uma agenda para a saúde. São Paulo: 
Hucitec; 2006.

25. Fleury S. A reforma sanitária e o SUS: questões de sustentabilidade. Cienc Saúde Col. 2007:12(2):307-17.

26. Jorge MAS. Engenho dentro de casa: sobre a construção de um serviço de atenção diária em saúde mental [dissertação]. Rio de Janeiro (RJ): Escola Nacional de Saúde Pública. Fundação Oswaldo Cruz; 1997.

27. Hirdes A, Kantorski LP. Sistematização do cuidado em enfermagem psiquiátrica. Texto Contexto Enferm. 2000; 9(2):94-105.

28. Pontes PAR, Fraga MNO. Reforma Psiquiátrica no Ceará: descrição de um caso. Rev Latino-Am Enferm. 1997; 5(especial):45-50.

29. Wetzel C. Avaliação de serviços de saúde mental: a construção de um processo participativo [tese]. Ribeirão Preto (SP): Escola de Enfermagem de Ribeirão Preto. Universidade de São Paulo; 2005.

30. Antunes SMMO, Queiroz MS. A configuração da reforma psiquiátrica em contexto local no Brasil: uma análise qualitativa. Cad Saúde Públ. 2007; 23(1):207-15. 